\title{
MICROLENSING OF LARGE SOURCES INCLUDING SHEAR TERM EFFECTS
}

S. REFSDAL

Hamburger Sternwarte

Gojenbergsweg 112, D-21029 Hamburg, Germany

AND

R. STABELL

Institute of Theoretical Astrophysics, University of Oslo

Pb. 1029, Blindern, N-0315 Oslo, Norway

Abstract. We find that the standard deviation for the observed magnitude of a large microlensed source is $\delta m \leq 2.17|\kappa|^{1 / 2} \theta_{\circ} / \theta_{s}$ even in the presence of non-zero shear.

\section{Introduction and Summary}

The most spectacular effects of microlensing occur when the angular radius of the source $\left(\theta_{s}\right)$ is much smaller than the Einstein Ring $\left(\theta_{0}\right)$, and this case has also been investigated in most detail up till now. For large sources $\left(\theta_{s} \geq 5 \theta_{0}\right)$ Refsdal and Stabell (1991) derived analytically a useful formula for the standard deviation of the observed magnitude:

$$
\delta m=2.17 \sqrt{|\kappa|} \frac{\theta_{\circ}}{\theta_{s}} .
$$

Here $\kappa$ is the optical depth for microlensing and the shear $\gamma$ is assumed to be zero. The variations in $m$ are mainly due to fluctuations in the smoothed out surface mass density caused by the Poisson fluctuations in the number of stars projected in front of the source. For $\kappa$-values equal to 0.1 and 0.4 the value of $\delta m$ given by Eq. (1) was found to be reasonably accurate (to within $20 \%$ ) for sources with $\theta_{s}>5 \theta_{0}$.

We have carried out more extensive calculations for various values of $\kappa, \theta_{s}$ and also for some values of $\gamma \neq 0$. The main result is that the value 


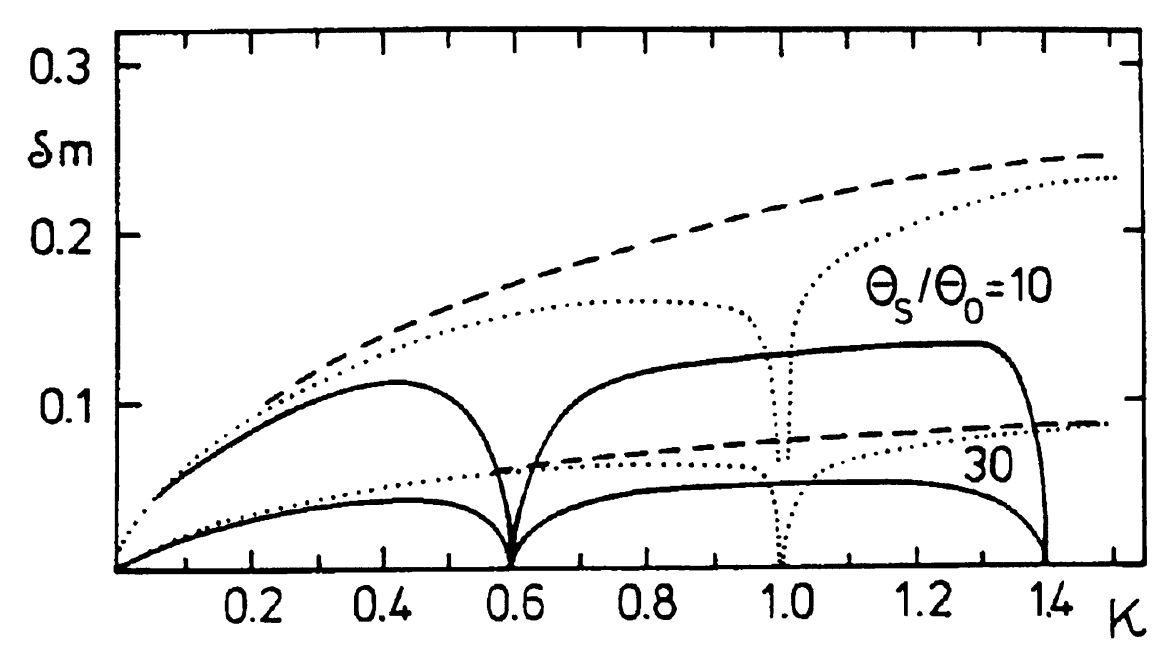

Figure 1. The standard deviation $\delta m$ for the case $\gamma=0.4$ together with the values of $\delta m$ given by Eq. (1) (dashed) and $\delta m$ for $\gamma=0$ (dotted)

of $\delta m$ given by Eq. (1) is an upper limit to the true standard deviation, regardless of the values of $\theta_{s} / \theta_{0}, \kappa$ and $\gamma$. This means that Eq. (1) still can be used to estimate an upper limit to the source size as discussed by Refsdal and Stabell (1991). As expected we find that the deviations from Eq. (1) increase with decreasing source size. Furthermore, we find that these deviations typically increase with decreasing value of $(1-\kappa)^{2}-\gamma^{2}$ (amplification increases, compare to Deguchi \& Watson (1987)).

\section{Results}

For the case $\gamma \neq 0$ we have not succeeded in deriving a simple analytical formula similar to Eq. (1). Some of the results from our numerical calculations are plotted in Fig. $1,\left(\theta_{s}=10 \theta_{\circ}\right.$ and $\theta_{s}=30 \theta_{\circ}$, and $\left.\gamma=0.4\right)$, together with results for $\gamma=0$ and the values of $\delta m$ given by Eq. (1).

It is generally found that even for $\gamma \neq 0$, Eq. (1) represents an upper limit for $\delta m$. Except for the narrow "forbidden" interval around $\kappa=1$, we even find that a value of $\gamma \neq 0$ further reduces $\delta m$. For a large range of $\gamma$-values we see however that the effect of the shear is rather small. An obvious exception is of course when $\gamma$ approaches $\pm(1-\kappa)$, since $\delta m$ then approaches zero.

\section{References}

Deguchi, S. \& Watson, W.D., 1987, Phys Rev Letters, 59, 2814

Refsdal, S. \& Stabell, R., 1991, A\&A, 250, 62 